\title{
A UNIFIED APPROACH TO SOME PREDICTION PROBLEMS
}

\author{
STEPHEN D. ABBOTT
}

(Communicated by Palle E. T. Jorgensen)

\begin{abstract}
In this paper we solve a general extremal problem for a nonnegative operator in Hilbert space. We show that it contains the classical infimum problems of Szegö and Kolmogorov for bounded weight functions on the circle and also prove some new prediction theorems.
\end{abstract}

\section{INTRODUCTION}

Let $\mathbf{C}$ be the complex numbers, $\mathbf{D}=\{z \in \mathbf{C}:|z|<1\}$, and $\partial \mathbf{D}=\{z \in$ $\mathrm{C}:|z|=1\}$. Define the function $\chi$ on $\partial \mathrm{D}$ by $\chi\left(e^{i \theta}\right)=e^{i \theta}$, and let $\sigma$ be the normalized Lebesgue measure on $\partial \mathbf{D}$. Given a nonnegative function $\omega\left(e^{i \theta}\right) \in L^{1}[\partial \mathbf{D}]$, we state two classical theorems from prediction theory:

(i) Szegö's infimum:

$$
\inf \left\{\int_{\partial \mathbf{D}}|1-\chi p|^{2} \omega d \sigma: p=\sum_{j=0}^{N} c_{j} \chi^{j}\right\}=\exp \left(\int_{\partial \mathbf{D}} \log \omega d \sigma\right) ;
$$

(ii) Kolmogorov's infimum:

$$
\inf \left\{\int_{\partial \mathbf{D}}|1-p|^{2} \omega d \sigma: p=\sum_{|j| \leq N} c_{j} x^{j}, \int_{\partial \mathbf{D}} p d \sigma=0\right\}=\left[\int_{\partial \mathbf{D}} \frac{1}{\omega} d \sigma\right]^{-1} .
$$

If the function appearing on the right-hand side of either equation above is not integrable, the infimum is understood to be zero.

We now pose an abstract problem that essentially contains both of these. Let $\mathscr{L}$ be a Hilbert space with inner product $\langle\cdot, \cdot\rangle$, and let $\mathscr{B}(\mathscr{L})$ be the set of bounded operators on $\mathscr{L}$. Let $P$ be the orthogonal projection onto a closed subspace $\mathscr{C} \subseteq \mathscr{L}$, and let $W$ be a nonnegative operator in $\mathscr{B}(\mathscr{L})$. Now fix $k \in \mathscr{C}$ and consider

$$
\inf \{\langle W(k-f),(k-f)\rangle: f \in \mathscr{L}, P f=0\} .
$$

Received by the editors December 18, 1992 and, in revised form, May 3, 1993.

1991 Mathematics Subject Classification. Primary 47B65, 47N30, 60G25; Secondary 47B35.

Key words and phrases. Szegö's infimum, Kolmogorov's infimum, prediction theory, Hardy space, Toeplitz operator, Hilbert space.

The author would like to acknowledge and thank his advisor, Professor Marvin Rosenblum, for many helpful suggestions and conversations. 
We can recover Kolmogorov's infimum via the identification $\mathscr{L}=L^{2}, \mathscr{C}=\mathrm{C}$, $k=1$, and $W=M_{\omega}$, which is multiplication by $\omega$. Note, however, that in this formulation $\omega$ must be bounded. For Szegö's infimum we let $\mathscr{L}$ be the Hardy space $H^{2}$ of the circle and $W$ be the Toeplitz operator $T_{\omega}$, which is multiplication by $\omega$ followed by the projection onto $H^{2}$. Here again $\omega$ must be bounded for $T_{\omega}$ to be in $\mathscr{B}\left(H^{2}\right)$.

In this paper we give a solution to the abstract extremal problem and then use it to derive the two classical results mentioned above. Because the hypothesis of the abstract result is so minimal, it is useful in solving many other prediction problems, and we explore some applications in the second half of this paper. For other generalizations of Szegö's infimum see [4, 5, 7]. Vector generalizations of Kolmogorov's infimum are also discussed in [7].

\section{A GENERAL PREDICTION THEOREM}

We will need the following fact.

Lemma 0. Let $\mathscr{M}$ be a closed subspace of a Hilbert space $\mathscr{L}$, and let $g \in \mathscr{L}$. Then

$$
\inf \{\|g+m\|: m \in \mathscr{M}\}=\sup \{|\langle g, l\rangle|: l \perp \mathscr{M},\|l\| \leq 1\}
$$

Proof. Let $P_{\mathscr{M}}$ be the projection onto $\mathscr{M}$ and $P_{\mathscr{M}}=I-P_{\mathscr{M}}$. Then

$$
\begin{aligned}
\inf \{\|g+m\|: m \in \mathscr{M}\} & =\left\|g-P_{\mathscr{M}} g\right\|=\left\|P_{\mathscr{M} \perp} g\right\| \\
& =\sup \left\{\left|\left\langle P_{\mathscr{M}} g, l\right\rangle\right|: l \in \mathscr{M}^{\perp},\|l\| \leq 1\right\} .
\end{aligned}
$$

To prove the main result we temporarily assume that the nonnegative operator $W$ is invertible in $\mathscr{B}(\mathscr{L})$. If $P$ is the projection onto a closed subspace $\mathscr{C}$ of $\mathscr{L}$, then the compression $A=P W^{-1} P$ is nonnegative and invertible in $\mathscr{B}(\mathscr{C})$ since for $c \in \mathscr{C},\langle A c, c\rangle=\left\langle W^{-1} c, c\right\rangle \geq \delta\|c\|^{2}$ for some $\delta>0$.

Theorem 1. Let $W$ be nonnegative and invertible in $\mathscr{B}(\mathscr{L})$, and let $P \in \mathscr{B}(\mathscr{L})$ be the projection onto a closed subspace $\mathscr{C} \subseteq \mathscr{L}$. Then for any $k \in \mathscr{C}$,

$$
\inf \{\langle W(k-f),(k-f)\rangle: P f=0\}=\left\langle\left[P W^{-1} P\right]^{-1} k, k\right\rangle .
$$

Proof. Set $\mathscr{M}=\left\{W^{1 / 2} f: P f=0\right\}$. Then

$$
\begin{aligned}
\inf \{ & \langle W(k-f),(k-f)\rangle: P f=0\}=\inf \left\{\left\|W^{1 / 2}(k-f)\right\|^{2}: P f=0\right\} \\
& =\inf \left\{\left\|W^{1 / 2} k-m\right\|^{2}: m \in \mathscr{M}\right\} \\
& =\left[\sup \left\{\left|\left\langle W^{1 / 2} k, l\right\rangle\right|: l \perp \mathscr{M},\|l\| \leq 1\right\}\right]^{2}
\end{aligned}
$$

by Lemma 0 . But $l \perp \mathscr{M}$ if and only if $W^{1 / 2} l \in \mathscr{C}$, so

$$
\begin{aligned}
& \sup \left\{\left|\left\langle W^{1 / 2} k, l\right\rangle\right|: l \perp \mathscr{M},|| l \| \leq 1\right\}=\sup \left\{\frac{\left|\left\langle W^{1 / 2} k, l\right\rangle\right|}{\|l\|}: W^{1 / 2} l \in \mathscr{C}\right\} \\
& \quad=\sup \left\{\frac{\left|\left\langle W^{1 / 2} k, W^{-1 / 2} c\right\rangle\right|}{\left\|W^{-1 / 2} c\right\|}: c \in \mathscr{C}\right\}=\sup \left\{\frac{|\langle k, c\rangle|}{\left\|W^{-1 / 2} c\right\|}: c \in \mathscr{C}\right\} .
\end{aligned}
$$

Recall that the operator $A=P W^{-1} P$ is nonnegative and invertible in $\mathscr{B}(\mathscr{C})$, and $\left\|W^{-1 / 2} c\right\|=\left\|A^{1 / 2} c\right\|$. Thus,

$$
\begin{gathered}
\sup \left\{\frac{|\langle k, c\rangle|}{\left\|W^{-1 / 2} c\right\|}: c \in \mathscr{C}\right\}=\sup \left\{\frac{\left|\left\langle A^{-1 / 2} k, A^{1 / 2} c\right\rangle\right|}{\left\|A^{1 / 2} c\right\|}: c \in \mathscr{C}\right\} \\
=\sup \left\{\left|\left\langle A^{-1 / 2} k, c\right\rangle\right|: c \in \mathscr{C},\|c\|=1\right\}=\left\|A^{-1 / 2} k\right\| .
\end{gathered}
$$


The last equality is true by observing that the supremum is attained when $c=$ $A^{-1 / 2} k /\left\|A^{-1 / 2} k\right\|$. Putting (1)-(3) together gives

$$
\begin{aligned}
& \inf \{\langle W(k-f),(k-f)\rangle: P f=0\} \\
& =\left\|A^{-1 / 2} k\right\|^{2}=\left\langle A^{-1} k, k\right\rangle=\left\langle\left[P W^{-1} P\right]^{-1} k, k\right\rangle .
\end{aligned}
$$

One attack on the case where the weight $W$ is not invertible is to consider the weights $W_{\varepsilon}=W+\varepsilon I$, where $\varepsilon>0$ and $I$ is the identity operator on $\mathscr{L}$.

Theorem 2. Let $W \in \mathscr{B}(\mathscr{L})$ be nonnegative, and let $P \in \mathscr{B}(\mathscr{L})$ be a projection. If $k \in P \mathscr{L}$, then

$$
\inf \{\langle W(k-f),(k-f)\rangle: P f=0\}=\lim _{\varepsilon \rightarrow 0}\left\langle\left[P W_{\varepsilon}^{-1} P\right]^{-1} k, k\right\rangle .
$$

Proof. Let $\beta=\inf _{P f=0}\langle W(k-f),(k-f)\rangle$ and

$$
\begin{aligned}
\beta_{\varepsilon} & =\inf _{P f=0}\left\langle W_{\varepsilon}(k-f),(k-f)\right\rangle \\
& =\inf _{P f=0}\left\{\langle W(k-f),(k-f)\rangle+\varepsilon\|k-f\|^{2}\right\} .
\end{aligned}
$$

The $\beta_{\varepsilon}$ 's are monotone decreasing with decreasing $\varepsilon$ and bounded below by $\beta$.

Let $\delta>0$ be arbitrary. Choose $f^{\prime}$ so that $P f^{\prime}=0$ and $\left\langle W\left(k-f^{\prime}\right)\right.$, $\left.\left(k-f^{\prime}\right)\right\rangle \leq \beta+\frac{\delta}{2}$. Next choose $\varepsilon_{0}$ so that $\varepsilon_{0}\left\|k-f^{\prime}\right\|^{2} \leq \frac{\delta}{2}$. Then

$$
\begin{aligned}
\beta_{\varepsilon_{0}} & =\inf _{P f=0}\left\{\langle W(k-f),(k-f)\rangle+\varepsilon_{0}\|k-f\|^{2}\right\} \\
& \leq\left\langle W\left(k-f^{\prime}\right),\left(k-f^{\prime}\right)\right\rangle+\varepsilon_{0}\left\|k-f^{\prime}\right\|^{2} \leq\left(\beta+\frac{\delta}{2}\right)+\frac{\delta}{2}=\beta+\delta .
\end{aligned}
$$

Hence, $\beta_{\varepsilon_{0}}-\beta \leq \delta$, which implies $\lim _{\varepsilon \rightarrow 0} \beta_{\varepsilon}=\beta$. The theorem follows from Theorem 1 .

\section{ApPlications}

We first show that Theorem 2 implies the Szegö and Kolmogorov results for bounded weight functions.

Example: Kolmogorov's infimum. Let $\omega\left(e^{i \theta}\right) \geq 0$ be in $L^{\infty}[\partial \mathrm{D}]$, and let $P f=$ $\int_{\partial \mathbf{D}} f d \sigma$ for $f \in L^{2}$. Then Theorem 2 with $\bar{k}=1$ asserts that

$$
\begin{gathered}
\inf \left\{\int_{\partial \mathbf{D}}|1-f|^{2} \omega d \sigma: f \in L^{2}, P f=0\right\}=\lim _{\varepsilon \rightarrow 0}\left\langle\left[P M_{\omega+\varepsilon}^{-1} P\right]^{-1} 1,1\right\rangle \\
\quad=\lim _{\varepsilon \rightarrow 0}\left[\int_{\partial \mathbf{D}} \frac{1}{\omega+\varepsilon} d \sigma\right]^{-1}=\left[\int_{\partial \mathbf{D}} \frac{1}{\omega} d \sigma\right]^{-1} .
\end{gathered}
$$

The last equality is from the monotone convergence theorem. If $\frac{1}{\omega}$ is not integrable then the limit is zero.

Example: Szegö's infimum. Let $\omega\left(e^{i \theta}\right) \geq \delta>0$ be in $L^{\infty}[\partial \mathrm{D}]$, and let

$$
g(z)=\exp \left[\int_{\partial \mathbf{D}} \frac{e^{i \theta}+z}{e^{i \theta}-z} \log \left(\frac{1}{\omega}\right)^{1 / 2} d \sigma\right], \quad z \in \mathbf{D} .
$$

Taking boundary values we get $1 / \omega\left(e^{i \theta}\right)=\left|g\left(e^{i \theta}\right)\right|^{2}$ a.e., where $g \in H^{\infty}$ and $g$ is outer [2, p. 67]. Let $T_{\omega}$ be the Toeplitz operator on $H^{2}$ with symbol $\omega$. 
Then $T_{\omega}^{-1}=T_{g} T_{g}[6$, p. 64]. We continue to let $P$ be the projection onto the constants and employ Theorem 1 to get

$$
\begin{aligned}
\inf \left\{\int_{\partial \mathbf{D}}|1-\chi f|^{2} \omega d \sigma: f \in H^{2}\right\} & =\left\langle\left[P T_{g} T_{\tilde{g}} P\right]^{-1} 1,1\right\rangle \\
& =\frac{1}{\left\langle T_{g} T_{\tilde{g}} 1,1\right\rangle}=\frac{1}{\left\|T_{\tilde{g}} 1\right\|^{2}} .
\end{aligned}
$$

But $\left\|T_{\bar{g}} 1\right\|^{2}=|g(0)|^{2}$ and $1 /|g(0)|^{2}=\exp \left[\int_{\partial \mathbf{D}} \log \omega d \sigma\right]$.

If $\omega$ is not bounded below, then Theorem 2 implies

$$
\inf _{f \in H^{2}}\left\{\int_{\partial \mathbf{D}}|1-\chi f|^{2} \omega d \sigma\right\}=\lim _{\varepsilon \rightarrow 0} \exp \left[\int_{\partial \mathbf{D}} \log (\omega+\varepsilon) d \sigma\right],
$$

and a monotone convergence argument gives the result.

Example: The Kolmogorov and Szegö infima in two variables. Let $\omega\left(e^{i \theta}, e^{i \phi}\right)$ be a nonnegative bounded function on $\partial \mathbf{D} \times \partial \mathbf{D}$. For this example let $L^{2}$ be the Hilbert space of square integrable functions on $\partial \mathbf{D} \times \partial \mathbf{D}$, and let $H^{2}$ be the analytic "quarter space" of $L^{2}$. That is, $f \in H^{2}$ if $f \in L^{2}$ and

$$
\int_{\partial \mathbf{D}} \int_{\partial \mathbf{D}} f\left(e^{i \theta}, e^{i \phi}\right) e^{i m \theta+i n \phi} d \sigma(\theta) d \sigma(\phi)=0,
$$

whenever $n$ or $m$ is positive. This is a closed subspace, and we let $P_{H^{2}}$ be the orthogonal projection from $L^{2}$ onto $H^{2}$. Finally, let $T_{\omega} \in \mathscr{B}\left(H^{2}\right)$ be the Toeplitz operator taking $f$ to $P_{H^{2}} \omega f$, and assume $T_{\omega}$ is invertible. Then Theorem 1 implies

$$
\begin{aligned}
\inf & \left\{\int_{\partial \mathbf{D}} \int_{\partial \mathbf{D}}|1-f|^{2} \omega d \sigma(\theta) d \sigma(\phi): f \in H^{2}, \int_{\partial \mathbf{D}} \int_{\partial \mathbf{D}} f d \sigma(\theta) d \sigma(\phi)=0\right\} \\
& =\frac{1}{\left\langle T_{\omega}^{-1} 1,1\right\rangle} .
\end{aligned}
$$

This is a bit unsatisfying since not much is known about $T_{\omega}^{-1}$, but it does reduce the task to the seemingly easier problem of evaluating $\left\langle T_{\omega}^{-1} 1,1\right\rangle$.

For Kolmogorov's infimum we have

$$
\begin{aligned}
\inf \left\{\int_{\partial \mathbf{D}} \int_{\partial \mathbf{D}}|1-f|^{2} \omega d \sigma(\theta) d \sigma(\phi): f \in L^{2}, \int_{\partial \mathbf{D}} \int_{\partial \mathbf{D}} f d \sigma(\theta) d \sigma(\phi)=0\right\} \\
\quad=\left[\int_{\partial \mathbf{D}} \int_{\partial \mathbf{D}} \frac{1}{\omega} d \sigma(\theta) d \sigma(\phi)\right]^{-1},
\end{aligned}
$$

and similar results hold in cases of more variables.

Example: A generalized Kolmogorov infimum. Returning to the one-variable setting, we again consider a nonnegative bounded function $\omega$ on $\partial \mathbf{D}$. Let $N$ be a nonnegative integer.

Proposition 3. If $\frac{1}{\omega} \in L^{1}$, then the matrix $\left[\alpha_{m, n}\right]_{m, n=-N}^{+N}$ given by $\alpha_{m, n}=$ $\int_{\partial \mathbf{D}} \chi^{n-m} \frac{1}{\omega} d \sigma$ is invertible, and for fixed $p=\sum_{|j| \leq N} c_{j} \chi^{j}$,

$$
\inf \left\{\int_{\partial \mathbf{D}}\left|p-\sum_{|j|>N} c_{j} \chi^{j}\right|^{2} \omega d \sigma: \sum_{|j|>N}\left|c_{j}\right|^{2}<\infty\right\}=\left\langle\left[\alpha_{m, n}\right]^{-1} p, p\right\rangle .
$$


Proof. Notice that $\operatorname{ker}\left[\alpha_{m, n}\right]=\{0\}$ since for any nonzero $p^{\prime}=\sum_{|j| \leq N} d_{j} \chi^{j}$,

$$
\left\langle\left[\alpha_{m, n}\right]\left[\begin{array}{c}
d_{-N} \\
\vdots \\
d_{N}
\end{array}\right],\left[\begin{array}{c}
d_{-N} \\
\vdots \\
d_{N}
\end{array}\right]\right\rangle=\int_{\partial \mathbf{D}} \frac{\left|p^{\prime}\right|^{2}}{\omega} d \sigma>0 .
$$

Thus $\left[\alpha_{m, n}\right]$ is invertible. Let $\varepsilon>0$, and let $P$ be the projection in $L^{2}$ onto $\bigvee\left\{\chi^{j}:|j| \leq N\right\}$. By Theorem 2,

$$
\inf \left\{\int_{\partial \mathbf{D}}\left|p-\sum_{|j|>N} c_{j} \chi^{j}\right|^{2} \omega d \sigma: \sum_{|j|>N}\left|c_{j}\right|^{2}<\infty\right\}=\lim _{\varepsilon \rightarrow 0}\left\langle\left[P M_{\omega+\varepsilon}^{-1} P\right]^{-1} p, p\right\rangle .
$$

Represent $P M_{\omega+\varepsilon}^{-1} P$ with the $(2 N-1) \times(2 N-1)$ matrix $\left[\alpha_{m, n}^{\varepsilon}\right]$ where $\alpha_{m, n}^{\varepsilon}=$ $\int_{\partial \mathbf{D}} \frac{1}{\omega+\varepsilon} \chi^{n-m} d \sigma$. Since $\frac{1}{\omega} \in L^{1}, \lim _{\varepsilon \rightarrow 0} \alpha_{m, n}^{\varepsilon}=\alpha_{m, n}$ and it follows that $\lim _{\varepsilon \rightarrow 0}\left\langle\left[\alpha_{m, n}^{\varepsilon}\right]^{-1} p, p\right\rangle=\left\langle\left[\alpha_{m, n}\right]^{-1} p, p\right\rangle$.

A similar problem is discussed in [3, p. 83].

Example: A Kolmogorov infimum for $2 \times 2$ matrix-valued weights. Let

$$
W\left(e^{i \theta}\right)=\left[\begin{array}{ll}
\omega_{11}\left(e^{i \theta}\right) & \omega_{12}\left(e^{i \theta}\right) \\
\omega_{12}\left(e^{i \theta}\right) & \omega_{22}\left(e^{i \theta}\right)
\end{array}\right]
$$

define a bounded nonnegative weight on $L^{2}$ functions from $\partial \mathbf{D}$ into $\mathbf{C}^{2}$. For $\varepsilon>0$, set $W_{\varepsilon}=W+\varepsilon I$. The function $\operatorname{det} W=\omega_{11} \omega_{22}-\left|\omega_{12}\right|^{2}$ is nonnegative and bounded, and the corresponding function $\operatorname{det} W_{\varepsilon}=\left(\omega_{11}+\varepsilon\right)\left(\omega_{22}+\varepsilon\right)-$ $\left|\omega_{12}\right|^{2}$ is bounded away from zero. Thus

$$
W_{\varepsilon}^{-1}\left(e^{i \theta}\right)=\frac{1}{\operatorname{det} W_{\varepsilon}\left(e^{i \theta}\right)}\left[\begin{array}{cc}
\omega_{22}\left(e^{i \theta}\right)+\varepsilon & -\omega_{12}\left(e^{i \theta}\right) \\
-\bar{\omega}_{12}\left(e^{i \theta}\right) & \omega_{11}\left(e^{i \theta}\right)+\varepsilon
\end{array}\right] .
$$

If $P$ is the projection onto the space of constants $\mathbf{C}^{2}$, then

$$
P W_{\varepsilon}^{-1} P=\left[\begin{array}{ll}
\alpha_{11}^{\varepsilon} & \alpha_{12}^{\varepsilon} \\
\bar{\alpha}_{12}^{\varepsilon} & \alpha_{22}^{\varepsilon}
\end{array}\right],
$$

where

$$
\alpha_{11}^{\varepsilon}=\int_{\partial \mathbf{D}} \frac{\omega_{22}+\varepsilon}{\operatorname{det} W_{\varepsilon}} d \sigma, \quad \alpha_{12}^{\varepsilon}=\int_{\partial \mathbf{D}} \frac{-\omega_{12}}{\operatorname{det} W_{\varepsilon}} d \sigma, \quad \alpha_{22}^{\varepsilon}=\int_{\partial \mathbf{D}} \frac{\omega_{11}+\varepsilon}{\operatorname{det} W_{\varepsilon}} d \sigma .
$$

Proposition 4. If $\frac{1}{\operatorname{det} W} \in L^{1}$ and $k \in \mathbf{C}^{2}$, then

$$
\inf \{\langle W(k-f),(k-f)\rangle: P f=0\}=\left\langle\left[\alpha_{i, j}\right]^{-1} k, k\right\rangle,
$$

where

$$
\alpha_{11}=\int_{\partial \mathbf{D}} \frac{\omega_{22}}{\operatorname{det} W} d \sigma, \quad \alpha_{12}=\int_{\partial \mathbf{D}} \frac{-\omega_{12}}{\operatorname{det} W} d \sigma, \quad \alpha_{22}=\int_{\partial \mathbf{D}} \frac{\omega_{11}}{\operatorname{det} W} d \sigma .
$$

Proof. Dominated convergence implies $\lim _{\varepsilon \rightarrow 0} \alpha_{i j}^{\varepsilon}=\alpha_{i j}$. By Theorem 2,

$$
\begin{aligned}
\inf _{P f=0}\langle W(k-f),(k-f)\rangle & =\lim _{\varepsilon \rightarrow 0}\left\langle\left[\alpha_{i j}^{\varepsilon}\right]^{-1} k, k\right\rangle \\
& =\lim _{\varepsilon \rightarrow 0}\left[\frac{1}{\alpha_{11}^{\varepsilon} \alpha_{22}^{\varepsilon}-\left|\alpha_{12}^{\varepsilon}\right|^{2}}\left\langle\left[\begin{array}{cc}
\alpha_{22}^{\varepsilon} & \alpha_{12}^{\varepsilon} \\
-\bar{\alpha}_{12}^{\varepsilon} & \alpha_{11}^{\varepsilon}
\end{array}\right] k, k\right\rangle\right] .
\end{aligned}
$$


We know a priori that this limit exists for all $k$, but before distributing the limit through we must make sure we do not have an indeterminate form. Choosing $k=\left[\begin{array}{l}1 \\ 0\end{array}\right]$ produces $\lim _{\varepsilon \rightarrow 0}\left(\alpha_{22}^{\varepsilon} /\left(\alpha_{11}^{\varepsilon} \alpha_{22}^{\varepsilon}-\left|\alpha_{12}^{\varepsilon}\right|^{2}\right)\right)$. Since the numerator does not go to zero, $\lim _{\varepsilon \rightarrow 0}\left(\alpha_{11}^{\varepsilon} \alpha_{22}^{\varepsilon}-\left|\alpha_{12}^{\varepsilon}\right|^{2}\right)>0$. Thus $\left[\alpha_{i j}\right]$ is invertible, and $\inf _{P f=0}\langle W(k-f),(k-f)\rangle=\left\langle\left[\alpha_{i j}\right]^{-1} k, k\right\rangle$.

Moving to the larger-dimensional case is more complicated because it is difficult to show, and may be false, that $\left[\alpha_{i j}\right]$ is invertible. Before doing one last application of Theorem 2 we state a short lemma back in the abstract Hilbert space setting.

Lemma 5. If $W \in \mathscr{B}(\mathscr{L})$ is nonnegative and $P \in \mathscr{B}(\mathscr{L})$ is a projection, then for any $k \in \mathscr{L}$

$$
\inf \{\langle W(k-f),(k-f)\rangle: P f=0\}=\inf \{\langle W(P k-f),(P k-f)\rangle: P f=0\} \text {. }
$$

Proof. Write $k=P k+k^{\prime}$, where $P k^{\prime}=0$. Then

$$
\begin{aligned}
\inf _{P f=0}\langle W(k-f),(k-f)\rangle & =\inf _{P f=0}\left\langle W\left(P k-\left(f-k^{\prime}\right)\right),\left(P k-\left(f-k^{\prime}\right)\right)\right\rangle \\
& =\inf _{P f=0}\langle W(P k-f),(P k-f)\rangle .
\end{aligned}
$$

Example: Unbiased estimation. Let $\left\{\psi_{j}\right\}_{j=0}^{N}$ be a set of linearly independent vectors in $L^{2}[\partial \mathbf{D}]$, and let $c_{j} \in \mathbf{C}$ for each $j$. Given a nonnegative function $\omega \in L^{\infty}$ and $k \in L^{2}$, we consider

$$
\inf \left\{\int_{\partial \mathbf{D}}|k+f|^{2} \omega d \sigma: f \in L^{2},\left\langle f, \psi_{j}\right\rangle=c_{j}, 0 \leq j \leq N\right\} .
$$

The approximating vectors do not lie in a subspace but rather are restricted by a set of linear functionals.

We first apply the Gram-Schmidt process to the vectors $\left\{\psi_{j}\right\}_{j=0}^{N}$. The algorithm for computing the new vectors and new values of the $c_{j}$ 's is straightforward [1, p. 25], so without loss of generality we proceed under the assumption that $\left\{\psi_{j}\right\}_{j=0}^{N}$ is an orthonormal set.

Proposition 6. If $\frac{1}{\omega} \in L^{\infty}$, then

$$
\begin{aligned}
\inf & \left\{\int_{\partial \mathbf{D}}|k+f|^{2} \omega d \sigma: f \in L^{2},\left\langle f, \psi_{j}\right\rangle=c_{j}, 0 \leq j \leq N\right\} \\
& =\left\langle\left[\alpha_{i, j}\right]^{-1}\left[\begin{array}{c}
a_{0} \\
\vdots \\
a_{N}
\end{array}\right],\left[\begin{array}{c}
a_{0} \\
\vdots \\
a_{N}
\end{array}\right]\right\rangle,
\end{aligned}
$$

where $\alpha_{i, j}=\int_{\partial \mathbf{D}}\left(\psi_{j} \bar{\psi}_{i} / \omega\right) d \sigma$ and $a_{j}=\left\langle k, \psi_{j}\right\rangle+c_{j}$.

Proof. If $P$ is the projection onto $\bigvee\left\{\psi_{j}: 0 \leq j \leq N\right\}$, then

$$
P f=\sum_{j=0}^{N}\left\langle f, \psi_{j}\right\rangle \psi_{j}
$$

for $f \in L^{2}$. Let $\mathscr{M}=P L^{2}$, and observe that the vector $p=\sum_{j=0}^{N} c_{j} \psi_{j}$ is the unique vector in $\mathscr{M}$ satisfying $\left\langle p, \psi_{j}\right\rangle=c_{j}$ for all $0 \leq j \leq N$. We now claim that

$$
\left\{f:\left\langle f, \psi_{j}\right\rangle=c_{j}, 0 \leq j \leq N\right\}=\left\{p+l: l \in L^{2}, P l=0\right\}
$$


To verify this, let $f \in L^{2}$ satisfy $\left\langle f, \psi_{j}\right\rangle=c_{j}$ for all $0 \leq j \leq N$. Then $P f$ also satisfies this condition, so $P f=p$. Thus $f=p+(I-P) f$ which gives one inclusion. The reverse inclusion follows from the straightforward calculation that $\left\langle p+l, \psi_{j}\right\rangle=c_{j}$ for all $0 \leq j \leq N$.

So we can rewrite the above infimum as

$$
\begin{aligned}
\inf \left\{\left\langle M_{\omega}(k+f),(k+f)\right\rangle:\left\langle f, \psi_{j}\right\rangle=c_{j}, 0 \leq j \leq N\right\} \\
\quad=\inf \left\{\left\langle M_{\omega}(k+p+l),(k+p+l)\right\rangle: P l=0\right\} \\
\quad=\inf \left\{\left\langle M_{\omega}(P k+p+l),(P k+p+l)\right\rangle: P l=0\right\},
\end{aligned}
$$

where the second equality is a consequence of Lemma 5 . By Theorem 2, this infimum is equal to

$$
\lim _{\varepsilon \rightarrow 0}\left\langle\left[P M_{\omega+\varepsilon}^{-1} P\right]^{-1}(P k+p),(P k+p)\right\rangle .
$$

Using $\left\{\psi_{j}\right\}_{j=0}^{N}$ as an orthonormal basis for $\mathscr{M}$, we have $P k+p=\sum_{j=0}^{N} a_{j} \psi_{j}$ where $a_{j}=\left\langle k, \psi_{j}\right\rangle+c_{j}$. We can also represent the operator $P M_{\omega+\varepsilon}^{-1} P$ with the matrix $\left[\alpha_{i, j}^{\varepsilon}\right]_{i, j=0}^{N}$, where $\alpha_{i, j}^{\varepsilon}=\int_{\partial \mathbf{D}} \psi_{j} \overline{\psi_{i}} \frac{1}{\omega+\varepsilon} d \sigma$. Since $\frac{1}{\omega} \in L^{\infty}$, dominated convergence implies $\lim _{\varepsilon \rightarrow 0} \alpha_{i, j}^{\varepsilon}=\alpha_{i, j}$. The matrix $\left[\alpha_{i, j}\right]_{i, j=0}^{N}$ must be invertible since for any nonzero $g \in \mathscr{M}$ we have $\left\langle\left[\alpha_{i, j}\right] g, g\right\rangle=\int_{\partial \mathbf{D}}\left(|g|^{2} / \omega\right) d \sigma>0$. Hence

$$
\lim _{\varepsilon \rightarrow 0}\left\langle\left[P M_{\omega+\varepsilon}^{-1} P\right]^{-1}(P k+p),(P k+p)\right\rangle=\left\langle\left[\alpha_{i, j}\right]^{-1}\left[\begin{array}{c}
a_{0} \\
\vdots \\
a_{N}
\end{array}\right],\left[\begin{array}{c}
a_{0} \\
\vdots \\
a_{N}
\end{array}\right]\right\rangle .
$$

The assumption $\frac{1}{\omega} \in L^{\infty}$ ensures that the integrals appearing above are finite. We could relax this to $\frac{1}{\omega} \in L^{1}$ and instead assume the $\psi_{j}$ 's are bounded.

\section{REFERENCES}

1. H. Dym and H. P. McKean, Fourier series and integrals, Academic Press, New York, 1972.

2. J. B. Garnett, Bounded analytic functions, Academic Press, New York, 1981.

3. U. Grenander and M. Rosenblatt, Statistical analysis of stationary time series, Wiley, New York, 1957.

4. B. Moore, The Szegö infimum, Proc. Amer. Math. Soc. 29 (1971), 55-62.

5. B. Moore, M. Rosenblum, and J. Rovnyak, Toeplitz operators associated with isometries, Proc. Amer. Math. Soc. 49 (1975), 189-194.

6. M. Rosenblum and J. Rovnyak, Hardy classes and operator theory, Oxford Univ. Press, New York, 1985.

7. S. R. Treil', Geometric methods in spectral theory of vector-valued functions: some recent results, Oper. Theory Adv. Appl., vol. 42, Birkhäuser, Basel, 1989, pp. 209-280.

Department of Mathematics and Computer Science, Middlebury College, MiddleBURY, VERMONT 05753

E-mail address: abbott@middlebury.edu 